\title{
Evaluation of the Anti-Diabetic and Anti-Ulcer Properties of Some Jordanian and Iraqi Medicinal Plants; a Screening Study
}

\author{
Husni A Twaij ${ }^{1}$ and Emad A S Al-Dujaili ${ }^{2}$ \\ ${ }^{1}$ Faculty of Pharmacy, Philadelphia University, Jordan \\ ${ }^{2}$ Dietetics, Nutrition and Biological Sciences, Queen Margaret University, Edinburgh, UK
}

Correspondence should be addressed to: Emad A S Al-Dujaili; ealdujaili@qmu.ac.uk

Received 22 October 2013; Accepted 27 January 2014; Published 19 February 2014

Academic Editor: Ozra Tabatabaei-Malazy

Copyright (C) 2014 Husni A Twaij and Emad A S Al-Dujaili. Distributed under Creative Commons CC-BY 3.0

\begin{abstract}
A large number of Jordanian and Iraqi medicinal plants have been used in traditional medicine for hypoglycemic and anti-ulcer activities. We have started a screening program to evaluate the activity of some of these plants and their extracts taking in consideration their use as an alternative therapy. Various parts of several plants were extracted and the hypoglycemic effects of the aqueous extracts have been examined in normal, glucose loaded and alloxan diabetic experimental animals. Teucrium polium aqueous extract was studied for its anti-ulcer activity of ulcers induced in rats by starvation and reserpine and/or stress. The aqueous extract of the aerial parts of Artemisia herba alba was administered orally to normoglycemic and alloxan diabetic rabbits and rats and found to produce a significant hypoglycemic activity (normoglycaemic: plasma glucose levels reduced from $116 \pm 4$ to $90 \pm 3 \mathrm{mg} / \mathrm{dL}$ after 90 minutes, $\mathrm{p}<0.001$; diabetic animals from $471 \pm 7$ to $399 \pm 9 \mathrm{mg} / \mathrm{dL}$, $\mathrm{p}<0.001$ ). The aerial aqueous extract of Phangnalon rupestre, Sinapis arvensis and Eryngium creticum also exhibited significant hypoglycemic effects in euglycaemic and glucose loaded rats. The other plants examined (Ditrichia graveolens, Alkanna strigosa, , Centaurea iberica, Asphodelin lutea, Paronychia argentea, rosmarinus officinalis, Achillea santolina, Achillea micrantha, Allium sativum, Centaurea phyllacephala, Centaurea behen, Crataegus azarolus, Prosopis farcta and Myrtus communis) failed to produce a significant change in the plasma glucose levels. One herb was comprehensively tested in detail for its anti-ulcer activity; Teucrium polium aqueous extract (intraperitoneally; $150 \mathrm{mg} / \mathrm{kg}$ ) produced an average of $50 \%$ healing of ulcers where as the orally administered extract produced $85 \%$ healing activity of ulcers when compared with the controls.
\end{abstract}

Keywords: Medicinal plants, Anti-diabetic, Anti-ulcer.

\section{Introduction}

Diabetes mellitus rely primarily on a life style changes and natural remedies may definitely offer a useful support to treat the disease. The present investigative project represents the up to date results obtained through a continuing programme related to the 
biological and biochemical screening of the local plants used in folk medicine. Throughout the ages, Arabian physicians have attempted the treatment of diabetes mellitus patients with indigenous plants (Said, 1969). Farnsworth and Segelman (1971) have described the activity of many herbs and plants that have also been pharmacologically proven to be of some value in diabetes mellitus (Lewis and ElvinLewis, 1977). Recently, primary prevention of diabetes mellitus has become necessary to control the huge spread of the disease to people of all ages as evidenced by several studies reported by Saravanamuttu (2012). It provides effective management and may prevent the onset of disease particularly in high-risk subjects. All of the plants tested under this study are commonly known by Arabic names, are popular folk remedy and claimed to be effective for the treatment of diabetes mellitus and some plants as antiulcer in Iraq and Jordan. For this purpose, the native populations have usually used a hot water decoction made from the fresh leaves and branches. The aim of this investigative study was to use the aqueous extracts of various plants in our experiments to mimic the procedure used in the traditional practices and report if there is really any effect on glucose levels. One plant extract was chosen to assess its anti-ulcer characteristics due to time and finance constraints.

\section{Materials and Methods}

The plants materials used in this study were collected in spring from the region of Iraq and Jordan. The samples were identified and authenticated by a specialist at the National Herbarium Department, Amman, Jordan. The aerial parts of the plants $(100 \mathrm{~g})$ were shade dried, pulverized and extracted with $500 \mathrm{~mL}$ of distilled water at room temperature overnight, then filtered and the filtrate evaporated in vacuum at $40^{\circ} \mathrm{C}$. The extracts obtained were dissolved in distilled water and administered to the animals following the proper dilutions to achieve the dose required.

\section{Animals}

Ethical approval for all the experiments performed was granted by the Faculty of Pharmacy, Philadelphia University Ethics Committee. Male adult Wistar rats (250-300 g), male adult Swiss albino mice (20-25 g) and male white New Zealand rabbits (1-2 kg) were used in the present studies. Animals described as fasted were deprived of food for at least 16 hours but allowed free access to tap water. The rats were anesthetized with sodium pentobarbitone, administered intraperitoneally at a dose of $40 \mathrm{mg} / \mathrm{kg}$. Collection of blood samples and administration of the materials to the rats were facilitated by the cannulated femoral arteries and veins respectively. Regarding the rabbits the marginal ear vein is used for administration of the materials and collection of blood samples. Alloxan diabetic animals were prepared by giving a dose of $150 \mathrm{mg} / \mathrm{kg}$ of freshly prepared alloxan monohydrate (BDH Chemicals). Glucose loads $(50 \% \mathrm{w} / \mathrm{v}, 4 \mathrm{~mL} / \mathrm{kg})$ were given to all animals $30 \mathrm{~min}$ following the plant extract or control vehicle administration.

\section{Effect on Plasma Glucose}

The animals were divided into 3 groups of eight animals each and were then given the following to each group respectively.

1. The aqueous extract of the plants under study.

2. The negative control (the vehicle solvent of the extracts).

3. The positive control (the reference drug Glyburide).

Blood samples were then taken just before the administration of the extracts and the controls and after 15, 30, 60 and 90 min post treatment. For the detailed Glucose load experiments, basal blood samples were taken at $-30 \mathrm{~min}$, just before the administration of the extracts and the controls (zero time) and after $30,60,90,120$, and 150 min there after. 


\section{Determination of the LD 50}

Increasing doses of the plant extracts were given to two groups of twelve mice. They were kept in transparent cages at $24 \mathrm{C}$. Mortality was recorded within 24 hours period (Litchfield and Wilcoxon, 1949).

\section{Behavioral Effects and Toxicity}

Gross behavioral studies were carried out in mice together with the LD50 studies including spontaneous motor activity and rectal temperature measurements. The animals were observed continuously for one hour, intermittently every 3 hours and then after 24 hours.

\section{Anti- Ulcer Activity}

Induction of ulcer: Normal white Wistar rats (300-350 g) of both sexes were used. The animal were subdivided into 2 groups, each animal was isolated in a separate cage with raised bottom and a wide wire mesh to prevent caprophagia. The animals were starved for 24 hours but water was permitted ad lib, then reserpine was administered $(20 \mathrm{mg} / \mathrm{kg}$, ip). The animals were then continued on starvation in the same cages for another 12 hours before sacrificing them after reserpine or vehicle treatments. The stomachs were removed, filled with $10 \mathrm{~mL}$ of $1 \%$ formalin in saline and then immersed in $10 \%$ formalin in saline, then each stomach was opened along the greater curvature and the mucosa was examined by the naked eye and under dissecting microscope. Ulcers were counted and measured to generate the ulcer index, which represent the total surface area of ulcer in $\mathrm{mm}^{2}$ per stomach. Representative ulcers were sampled for histopathological examination, processed by conventional technique using the Shandon automatic processor, and stained by hematoxylin and eosin. Sections were used to assess the depth of ulcers.

\section{Treatment of the Induced UIcer}

Aqueous extract of T. polium (150mg/kg i.p.), saline $(1 \mathrm{ml})$ and Cimitidine $20 \mathrm{mg} / \mathrm{kg}$ subcutaneously were given three times as follow; 120 min before, 15 min and $120 \mathrm{~min}$ after reserpine administration. The twelve hours period starvation was then continued as above. Afterwards the animals were allowed for normal housing with food ad lib for 48 hours during which T.polium aqueous extract $(150 \mathrm{mg} / \mathrm{kg})$ or controls were given orally twice daily (Twaij and Al-Badr, 1988). The ulcers were assessed as above and the degree of healing was determined accordingly and reported as percentage in comparison with the untreated animals.

\section{Statistical Methods}

All data collected was first stored using Microsoft Excel. A two tailed paired t-test was used first to determine any significant difference between baseline and intervention. Statistical analysis was then performed using One-Way ANOVA in SPSS software for Windows version 17.0 and using Student's T-tests in Microsoft Excel 2010. All data is expressed as Mean \pm Standard deviation or Error of the Mean ( \pm SD or SEM) unless otherwise stated. $\mathrm{P} \leq 0.05$ was considered statistically significant for all tests.

\section{Results}

\section{Behavioral Effects and Acute Toxicity}

LD50 and extract's yield for the plants investigated are shown in Table 1. All of the extracts under study had no significant changes in the spontaneous motor activity and rectal temperature. They did not produce any other changes in behaviour, food and water intake and the morphology of viscera (Twaij et al, 1987). 
Table 1: LD50 and Extract's Yield for the Plants Investigated. Name in Brackets is the Plant Family

\begin{tabular}{|l|l|l|l|}
\hline Scientific name & Local name & \% Yield & LD50 \\
\hline $\begin{array}{l}\text { Asphodelin Lutea } \\
\text { (Liliaceae) }\end{array}$ & Abu Swai & 12.37 & $\begin{array}{l}6800 \mathrm{mg} / \mathrm{kg} \text { equivalent to } \\
54.97 \mathrm{~g} \text { crude }\end{array}$ \\
\hline $\begin{array}{l}\text { Eryngium Creticum Lam } \\
\text { (Umbelliferaceae) }\end{array}$ & Shuk al-Akrabati & 10.08 & $\begin{array}{l}2400 \mathrm{mg} / \mathrm{kg} \text { equivalent to } \\
23.8 \mathrm{~g} \text { crude }\end{array}$ \\
\hline $\begin{array}{l}\text { Paronychia Argentea } \\
\text { (Caryophyllaceae) }\end{array}$ & Regel al-Hamama & 12.5 & $\begin{array}{l}420 \mathrm{mg} / \mathrm{kg} \text { equivalent to } \\
3.36 \mathrm{~g} \mathrm{crude}\end{array}$ \\
\hline Rosmarinus Officinalis & Ekleel-elbahar & 14.0 & $\begin{array}{l}55.86 \mathrm{~g} / \mathrm{kg} \text { of the crude } \\
\text { powder }\end{array}$ \\
\hline Centaurea phyllacephala & & 12.56 & $\begin{array}{l}2050 \mathrm{mg} / \mathrm{kg} \text { equivalent to } \\
33.8 \mathrm{~g} \mathrm{crude}\end{array}$ \\
\hline Centaurea behen & & 11.44 & $\begin{array}{l}2010 \mathrm{mg} / \mathrm{kg} \text { equivalent to } \\
25.8 \mathrm{~g} \mathrm{crude}\end{array}$ \\
\hline Crataegus & EL-Nabeq & 10.82 & $\begin{array}{l}3200 \mathrm{mg} / \mathrm{kg} \text { equivalent to } \\
43.8 \mathrm{~g} \text { crude }\end{array}$ \\
\hline Teucrium polium & & 13.6 & $380 \mathrm{mg} / \mathrm{kg}$ equivalent to \\
& & & $3.46 \mathrm{~g} \mathrm{crude}$ \\
\hline
\end{tabular}

\section{Anti-diabetic and Hypoglycemic Study}

The results obtained from the present studies showed that the oral administration of the aerial parts of Artemisea herba alba to glucose-fed, and to alloxan-induced diabetic rats and rabbits produced significant hypoglycemic activity (Twaij et al, 1987a). The aerial aqueous extract of both Phangnalon rupestre and Eryngium creticum also exhibited a potent and significant hypoglycemic effects in normal-fed and glucose loaded rats (Twaij and A-Jaleel, 2002; A-Jaleel and Twaij, 2002). In contrast to the above mentioned positive effects, all the following plant extracts (Ditrichia graveolens, Alkanna strigosa, Sinapis arvensis, Centaurea iberica,
Asphodelin lutea, Paronychia argentea, rosmarinus officinalis, Achillea santolina, Achillea micrantha, Allium sativum, Centaurea phyllacephala, Centaurea behen, Crataegus azarolus failed to produce a significant hypoglycemic or hyperglycemic effects. Some produced slight hyperglycemic effects or mild elevation in the plasma glucose levels (AI-Khazarji et al, 1993; Twaij et al, 1989; Al-Shamaony et al, 1994; Twaij et al, 1983; Twaij et al, 1987b; Twaij et al, 1988a \& b), in comparison with effects obtained from the controls. On the other hand, Myrtus communis and Prosopis farcta produced a significant hyperglycemic activity at 90 min compared to zero time. See Tables 2 and 3 . 
Table 2: Effect of the Aqueous Extract of Various Types of Plant on the Plasma Level of glucose (mg/dL) in Normal, Glucose Load and Alloxan Diabetic Rats in Comparison with their Vehicle Solvent (Saline) in Control Rats (Values are Mean \pm sem)

\begin{tabular}{|c|c|c|c|c|c|}
\hline \multirow{2}{*}{$\begin{array}{l}\text { Name of the plants and } \\
\text { their Controls } \\
\end{array}$} & \multirow{2}{*}{$\begin{array}{l}\text { Pretreatment } \\
\text { Values } \\
0\end{array}$} & \multicolumn{4}{|c|}{ Post treatment values (min.) } \\
\hline & & 15 & 30 & 60 & 90 \\
\hline 1. Artemisea herba alba & $116 \pm 4$ & $113 \pm 4$ & $105 \pm 4$ & $97 \pm 3 *$ & $90 \pm 3 * *$ \\
\hline Saline & $125 \pm 1$ & $128.5 \pm 1$ & $129.5 \pm 4$ & $128 \pm 3$ & $128 \pm 3$ \\
\hline $\begin{array}{l}\text { Artemisea herba.alba } \\
\text { Alloxan(Solvent) }\end{array}$ & $452.5 \pm 4$ & $459 \pm 3$ & $460.5 \pm 3$ & $462 \pm 3$ & $457 \pm 2$ \\
\hline $\begin{array}{l}\text { Artemisea herba alba } \\
\text { Alloxan(diabetic) }\end{array}$ & $471 \pm 7$ & $456 \pm 7$ & $437 \pm 8$ & $416 \pm 10^{*}$ & $399 \pm 9 *$ \\
\hline 2. Crataegus azarolus & $104 \pm 4$ & $109 \pm 4$ & $108 \pm 5$ & $111 \pm 4$ & $110 \pm 5$ \\
\hline Solvet & $104 \pm 2$ & $108 \pm 3$ & $113 \pm 2$ & $106 \pm 4$ & $110 \pm 4$ \\
\hline 3. Allium sativum & $93 \pm 5$ & $99 \pm 9$ & $99 \pm 7$ & $96 \pm 5$ & $95 \pm 4$ \\
\hline Solvent & $84 \pm 4$ & $88 \pm 4$ & $82 \pm 4$ & $90 \pm 6$ & $89 \pm 3$ \\
\hline 4. Myrtus communis & $100 \pm 3$ & $107 \pm 3$ & $115 \pm 2$ & $98 \pm 3$ & $98 \pm 2$ \\
\hline Solvent & $95 \pm 2$ & $105 \pm 3$ & $99 \pm 2$ & $90 \pm 3$ & $92 \pm 2$ \\
\hline $\begin{array}{l}\text { Myrtus communis } \\
\text { On glucose load }\end{array}$ & $82 \pm 2$ & $132 \pm 2$ & $152 \pm 6$ & $150 \pm 2$ & $145 \pm 5^{*}$ \\
\hline Solvent & $81 \pm 2$ & $131 \pm 2$ & $117 \pm 2$ & $91 \pm 3$ & $95 \pm 4$ \\
\hline 5. Prosopis farcta & $121 \pm 7$ & $217 \pm 19$ & $221 \pm 14$ & $233 \pm 25$ & $244 \pm 24^{*}$ \\
\hline Solvent & $116 \pm 4$ & $127 \pm 7$ & $120 \pm 8$ & $120 \pm 8$ & $121 \pm 8$ \\
\hline $\begin{array}{l}\text { 6. Centaurea phylloceph } \\
\text {-ala }\end{array}$ & $95 \pm 6$ & $115 \pm 4$ & $125 \pm 5$ & $99 \pm 5$ & $100 \pm 6$ \\
\hline Solvent & $100 \pm 8$ & $101 \pm 6$ & $108 \pm 8$ & $107 \pm 5$ & $105 \pm 6$ \\
\hline $\begin{array}{l}\text { Centaurea Phylloceph } \\
\text {-ala + glucose load }\end{array}$ & $111 \pm 5$ & $121 \pm 4$ & $117 \pm 4$ & $109 \pm 5$ & $110 \pm 6$ \\
\hline solvent + glucose load & $116 \pm 4$ & $120 \pm 7$ & $119 \pm 6$ & $120 \pm 6$ & $121 \pm 4$ \\
\hline 7. Centaurea behen & $105 \pm 6$ & $139 \pm 10$ & $123 \pm 12$ & $106 \pm 5$ & $115 \pm 7$ \\
\hline Solvent & $107 \pm 6$ & $106 \pm 4$ & $108 \pm 5$ & $109 \pm 6$ & $110 \pm 5$ \\
\hline 8. Achillea santolina & $115 \pm 5$ & $160 \pm 3$ & $159 \pm 5$ & $112 \pm 5$ & $115 \pm 4$ \\
\hline Solvent & $107 \pm 3$ & $104 \pm 6$ & $102 \pm 6$ & $107 \pm 9$ & $109 \pm 6$ \\
\hline $\begin{array}{l}\text { 9. Achillea } \\
\text { micrantha }\end{array}$ & $106 \pm 2$ & $121 \pm 4$ & $131 \pm 3$ & $116 \pm 4$ & $111 \pm 5$ \\
\hline
\end{tabular}


Table 3: Effect of the Orally Given Aqueous Extracts of Tested Herbs on Blood Glucose Concentration, before and after an Oral Glucose Load $(4 \mathrm{ml} / \mathrm{kg} \mathrm{of} 50 \% \mathrm{w} / \mathrm{v})$ in Comparison with their Negative (DW. Water) and Positive (Glyburide $5 \mathrm{mg} / \mathrm{kg}$ ) Controls when Given to Fasted Rats. Values are Mean \pm SEM, $N=9$.

(A) Ditrichia Graveolens, Alkanna Strigosa, Phagnalon Rupestre, Sinapis Arvensis, Centaurea Iberica

\begin{tabular}{|c|c|c|c|c|c|c|c|}
\hline \multirow{3}{*}{$\begin{array}{l}\text { Name of plants } \\
\text { And their } \\
\text { controls }\end{array}$} & \multicolumn{7}{|c|}{ Blood glucose concentration $\mathrm{mg} / \mathrm{dL}$} \\
\hline & $\begin{array}{l}\text { Pre-Treatment } \\
\text { and Time of }\end{array}$ & $\begin{array}{l}\text { After } \\
\text { treatment and }\end{array}$ & \multicolumn{5}{|c|}{ After glucose administration } \\
\hline & $-30 \mathrm{~min}$ & 0 time & $30 \mathrm{~min}$ & $60 \mathrm{~min}$ & $90 \min$ & $120 \mathrm{~min}$ & $150 \mathrm{~min}$ \\
\hline $\begin{array}{l}\text { I. a. Ditrichia } \\
\text { graveolens }\end{array}$ & $90.13 \pm 1.47$ & $\begin{array}{l}101.75 \\
\pm 1.51\end{array}$ & $\begin{array}{l}163.5 \\
\pm 1.96\end{array}$ & $\begin{array}{l}138.75 \\
\pm 1.86\end{array}$ & $\begin{array}{l}107.63 \\
\pm 0.96^{*}\end{array}$ & $\begin{array}{l}113.0 \\
\pm 1.09\end{array}$ & $\begin{array}{l}105.25 \\
\pm 0.98\end{array}$ \\
\hline b.D. Water & $89.38 \pm 1.53$ & $\begin{array}{r}99.88 \\
\pm 1.86\end{array}$ & $\begin{array}{l}163.95 \\
\pm 1.55\end{array}$ & $\begin{array}{l}129.63 \\
\pm 2.47\end{array}$ & $\begin{array}{l}112.13 \\
\pm 1.62\end{array}$ & $\begin{array}{l}104.38 \\
\pm 1.43\end{array}$ & $\begin{array}{l}91.5 \\
\pm 0.73\end{array}$ \\
\hline Glibenclamide & $91.25 \pm 1.75$ & $\begin{array}{l}101.38 \\
\pm 2.39 \\
\end{array}$ & $\begin{array}{l}155.63 \\
\pm 2.68 \\
\end{array}$ & $\begin{array}{l}126.13 \\
\pm 2.59 \\
\end{array}$ & $\begin{array}{l}94.75 \\
\pm 1.95 \\
\end{array}$ & $\begin{array}{l}73.13 \\
\pm 1.00 \\
\end{array}$ & $65.38 \pm 1.89$ \\
\hline $\begin{array}{l}\text { II a.Alkanna } \\
\text { Strigosa }\end{array}$ & $91.5 \pm 1.43$ & $\begin{array}{l}100.38 \\
\pm 1.15 \\
\end{array}$ & $\begin{array}{l}164.38 \\
\pm 2.01 \\
\end{array}$ & $\begin{array}{l}139.75 \\
\pm 0.80\end{array}$ & $\begin{array}{l}119.63 \\
\pm 0.63 \\
\end{array}$ & $\begin{array}{l}107.0 \\
\pm 1.16 \\
\end{array}$ & $\begin{array}{l}99.0 \\
\pm 0.94 \\
\end{array}$ \\
\hline b.D Water & $94.63 \pm 1.56$ & $101.25 \pm 1.0$ & $\begin{array}{l}155.88 \\
\pm 1.16 \\
\end{array}$ & $\begin{array}{l}130.25 \\
\pm 1.03\end{array}$ & $113.63 \pm 1.6$ & $104.5 \pm 0.87$ & $92.38 \pm 0.86$ \\
\hline Glibenclamide & & $100.0 \pm 1.85$ & $\begin{array}{l}154.88 \\
\pm 1.63 \\
\end{array}$ & $121.5 \pm 1.4$ & $97.0 \pm 2.16$ & $74.38 \pm 2.00$ & $64.00 \pm 1.43$ \\
\hline $\begin{array}{ll}\text { III } & \text {.a } \\
\text {.Phagnalon } \\
\text { rupestre }\end{array}$ & $93.75 \pm 2.12$ & $99.38 \pm 2.05$ & $163.5 \pm 5.22$ & $116.25 \pm 3.63^{* * *}$ & $94.63 \pm 2.12^{* * *}$ & $84.88 \pm 1.91$ & $80.00 \pm 1.44^{* * *}$ \\
\hline b. D. Water & $90.38 \pm 1.75$ & $97.38 \pm 1.59$ & $\begin{array}{l}158.75 \\
\pm 4.42\end{array}$ & $\begin{array}{l}134.13 \\
\pm 3.80\end{array}$ & $116.38 \pm 2.4$ & $\begin{array}{l}103.38 \\
\pm 2.39\end{array}$ & $94.38 \pm 1.68$ \\
\hline Glibenclamide & $91.50 \pm 1.93$ & $\begin{array}{l}102.78 \\
\pm 2.47 \\
\end{array}$ & $\begin{array}{l}157.75 \\
\pm 3.82 \\
\end{array}$ & $118.0 \pm 2.69$ & $91.63 \pm 1.84$ & $68.75 \pm 1.15$ & $61.50 \pm 1.94$ \\
\hline $\begin{array}{l}\text { IV a. Sinapis } \\
\text { arvensis }\end{array}$ & $91.38 \pm 1.31$ & $104.88 \pm 1.9$ & $\begin{array}{l}178.63 \\
\pm 2.28\end{array}$ & $145 . \pm 5.06$ & $\begin{array}{l}116.63 \\
\pm 2.23\end{array}$ & $\begin{array}{l}104.88 \\
\pm 0.83\end{array}$ & $88.5 \pm 1.96^{*}$ \\
\hline b. D. Water & $92.5 \pm 0.98$ & $100.5 \pm 2.15$ & $\begin{array}{l}170.88 \\
\pm 3.26\end{array}$ & $\begin{array}{l}137.5 \\
\pm 1.88\end{array}$ & $\begin{array}{l}123.25 \\
\pm 1.93\end{array}$ & $106.75 \pm 1.3$ & $94.38 \pm 1.79$ \\
\hline Glibenclamide & $91.88 \pm 1.26$ & $\begin{array}{l}103.88 \\
\pm 1.77 \\
\end{array}$ & $\begin{array}{l}154.25 \\
\pm 3.04 \\
\end{array}$ & $\begin{array}{l}107.88 \\
\pm 2.54 \\
\end{array}$ & $\begin{array}{l}84.63 \\
\pm 2.53 \\
\end{array}$ & $68.75 \pm 1.15$ & $59.38 \pm 2.47$ \\
\hline $\begin{array}{l}\text { V.a. Centaurea } \\
\text { Iberica }\end{array}$ & $\begin{array}{l}91.75 \\
\pm 1.37 \\
\end{array}$ & $\begin{array}{l}103.00 \\
\pm 3.04 \\
\end{array}$ & $\begin{array}{l}172.75 \\
\pm 4.75 \\
\end{array}$ & $\begin{array}{l}149.25 \\
\pm 2.52\end{array}$ & $\begin{array}{l}138.38 \\
\pm 3.42 \\
\end{array}$ & $\begin{array}{l}124.25 \\
\pm 2.48\end{array}$ & $111.00 \pm 2.5$ \\
\hline b. D. Water & $91.88 \pm 1.49$ & $97.13 \pm 1.49$ & $\begin{array}{l}160.5 \\
\pm 5.19\end{array}$ & $\begin{array}{l}138.5 \\
\pm 3.65\end{array}$ & $\begin{array}{l}121.75 \\
\pm 2.55\end{array}$ & $\begin{array}{l}108.75 \\
\pm 3.15\end{array}$ & $98.13 \pm 2.08$ \\
\hline Glibenclamide & $93.13 \pm 1.77$ & $\begin{array}{l}102.63 \\
\pm 2.23\end{array}$ & $\begin{array}{l}155.25 \\
\pm 5.20\end{array}$ & $\begin{array}{l}117.38 \\
\pm 5.71\end{array}$ & $89.63 \pm 3.00$ & $65.75 \pm 1.74$ & $58.75 \pm 1.69$ \\
\hline
\end{tabular}


(B) Asphodeline Lutea, Eryngium Creticum, Paronychia Argentea and Rosmarinus Officinalis

\begin{tabular}{|c|c|c|c|c|c|c|c|}
\hline \multirow{3}{*}{$\begin{array}{l}\text { Name of plants And } \\
\text { their controls }\end{array}$} & \multicolumn{7}{|c|}{ Blood glucose concentration $\mathrm{mg} / \mathrm{dL}$} \\
\hline & \multirow{2}{*}{$\begin{array}{l}\text { Pre-Treatment } \\
\text { and Time of } \\
\text { drug } \\
\text { administration } \\
\text { - } 30 \mathrm{~min}\end{array}$} & \multirow{2}{*}{$\begin{array}{l}\text { After } \\
\text { treatment and } \\
\text { time of glucose } \\
\text { load } \\
\text { administration } \\
\text { O time }\end{array}$} & \multicolumn{5}{|c|}{ After glucose administration } \\
\hline & & & $30 \mathrm{~min}$ & $60 \mathrm{~min}$ & $90 \mathrm{~min}$ & $120 \mathrm{~min}$ & $150 \mathrm{~min}$ \\
\hline $\begin{array}{l}\text { VI. a. Asphodelin } \\
\text { Lutea }\end{array}$ & $92 \pm 2.71$ & $102.13 \pm 1.57$ & $160.13 \pm 5.3$ & $147.5 \pm 4.07$ & $124.75 \pm 3.51$ & $\begin{array}{l}114.75 \\
\pm 2.44 \\
\end{array}$ & $\begin{array}{l}103.38 \\
\pm 2.97 \\
\end{array}$ \\
\hline b.D. Water & $88.13 \pm 1.16$ & $103.25 \pm 1.59$ & $\begin{array}{l}147.13 \\
\pm 2.85\end{array}$ & $\begin{array}{l}147.13 \\
\pm 3.99\end{array}$ & $123.38 \pm 2.38$ & $\begin{array}{l}106.25 \\
\pm 1.58 \\
\end{array}$ & $94.75 \pm 0.84$ \\
\hline c. Glibenclamide & $91.88 \pm 1.30$ & $100.25 \pm 1.58$ & $\begin{array}{l}158.85 \\
\pm 4.42\end{array}$ & $\begin{array}{l}117.75 \\
\pm 1.55\end{array}$ & $96.00 \pm 2.83$ & $64.88 \pm 3.65$ & ND \\
\hline $\begin{array}{l}\text { VII .a.Eryngium } \\
\text { certicum }\end{array}$ & $92.0 \pm 1.25$ & $106.5 \pm 2.20$ & $152.25 \pm 3.37$ & $\begin{array}{l}115.88 \\
\pm 1.58 * * * \\
\end{array}$ & $\begin{array}{l}101.13 \\
\pm 2.48 * * * \\
\end{array}$ & $\begin{array}{l}91.00 \\
\pm 2.15 * * * \\
\end{array}$ & $\begin{array}{l}86.13 \\
\pm 1.30 * * * \\
\end{array}$ \\
\hline b.D Water & $91.38 \pm 1.58$ & $102.63 \pm 2.02$ & $\begin{array}{l}165.88 \\
\pm 4.87\end{array}$ & $\begin{array}{l}143.38 \\
\pm 4.21 \\
\end{array}$ & $121.63 \pm 3.05$ & $106.5 \pm 1.91$ & $95.75 \pm 1.25$ \\
\hline c. Glibenclamide & $90.75 \pm 1.71$ & $99.50 \pm 2.35$ & $\begin{array}{l}157.25 \\
\pm 4.92 \\
\end{array}$ & $\begin{array}{l}122.63 \\
\pm 2.79 \\
\end{array}$ & $95.75 \pm 1.68$ & $71.00 \pm 1.95$ & ND \\
\hline $\begin{array}{l}\text { VIII .a Paronychia } \\
\text { argentea }\end{array}$ & $91.25 \pm 1.35$ & $105.75 \pm 1.78$ & $\begin{array}{l}171.38 \\
\pm 4.86 \\
\end{array}$ & $137.75 \pm 3.41$ & $133.63 \pm 2.43$ & $\begin{array}{l}118.63 \\
\pm 4.06 \\
\end{array}$ & $\begin{array}{l}114.88 \\
\pm 3.25 \\
\end{array}$ \\
\hline b. D. Water & $88.13 \pm 1.56$ & $103.25 \pm 1.28$ & $172.5 \pm 3.09$ & $\begin{array}{l}140.13 \\
\pm 2.64 \\
\end{array}$ & $125.63 \pm 0.94$ & $101.0 \pm 1.07$ & $91.25 \pm 0.90$ \\
\hline c. Glibenclamide & $88.13 \pm 1.53$ & $99.63 \pm 2.27$ & $152 \pm 3.15$ & $115.0 \pm 2.01$ & $91.13 \pm 1.79$ & $74.25 \pm 1.75$ & $65.38 \pm 2.23$ \\
\hline $\begin{array}{l}\text { IX a. Rosmarinus } \\
\text { officinslis }\end{array}$ & $94.5 \pm 1.08$ & $103.25 \pm 1.08$ & $\begin{array}{l}162.25 \\
\pm 3.08\end{array}$ & $128.75 \pm 2.3$ & $103.13 \pm 2.17$ & $\begin{array}{l}100.88 \\
\pm 2.57\end{array}$ & $\begin{array}{l}100.88 \\
\pm 2.57\end{array}$ \\
\hline b. D. Water & $92.38 \pm 0.91$ & $102 . \pm 1.21$ & $\begin{array}{l}185.86 \\
\pm 3.56\end{array}$ & $\begin{array}{l}147.38 \\
\pm 2.96\end{array}$ & $111.5 \pm 2.39$ & $101.75 \pm 1.8$ & $\begin{array}{l}111.13 \pm 2.4 \\
0\end{array}$ \\
\hline c. Glibenclamide & $92.25 \pm 1.10$ & $101.25 \pm 1.16$ & $156.38 \pm 1.92$ & $122.5 \pm 1.65$ & $97.88 \pm 1.29$ & $67.38 \pm 1.64$ & ND \\
\hline
\end{tabular}

\section{Anti-Ulcer Study}

The aqueous extract of Teucrium polium $(150 \mathrm{mg} / \mathrm{kg}$ IP) produced $50 \%$ healing of ulcers while the oral administration of the extract produced $85 \%$ healing activity of the ulcers. These results were statistically significant when compared with the saline and cimetidine in control animals (Twaij and Al-Badr, 1988). See figure 1. 


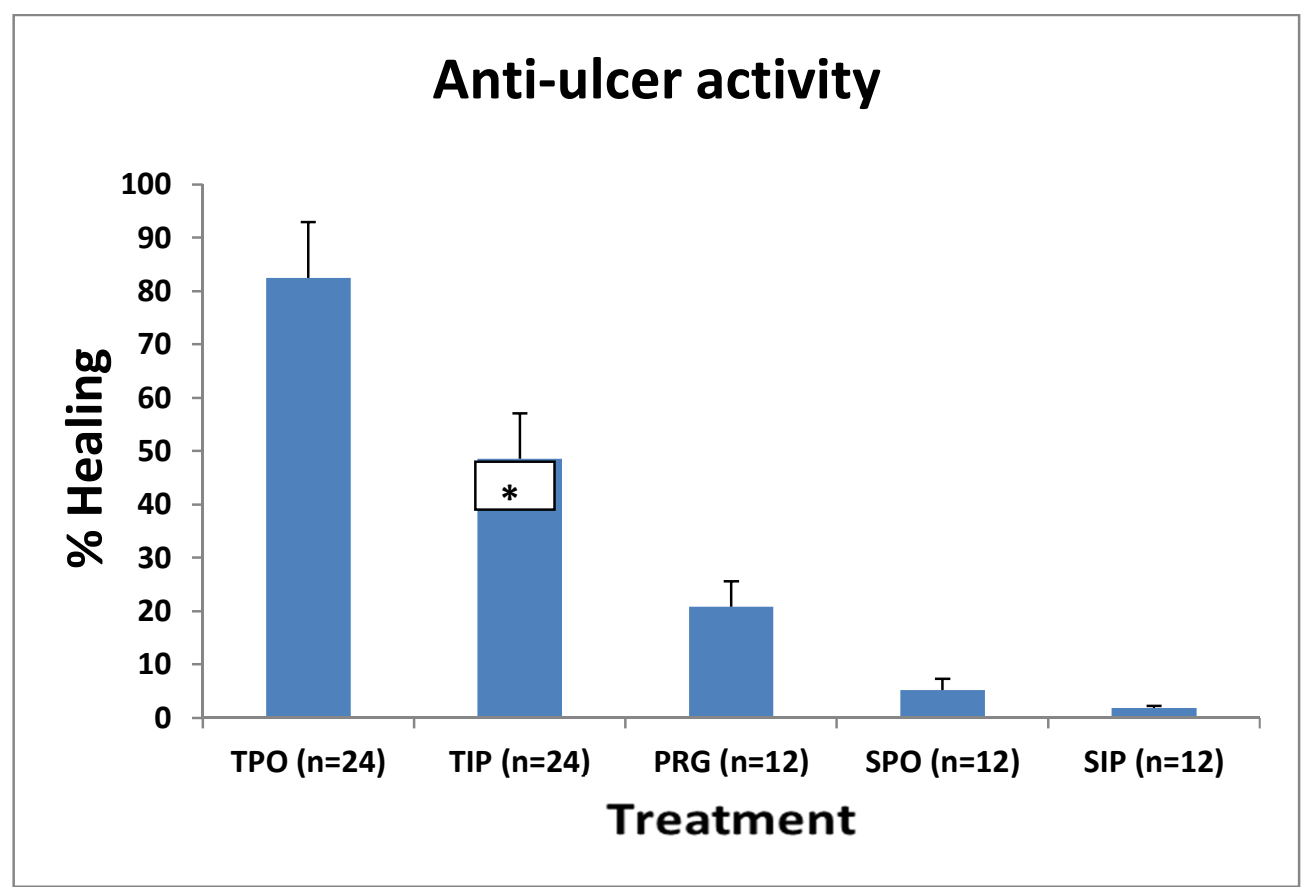

Figure 1. Effect of Teucrium Polium Aqueous Extract Given Orally at $150 \mathrm{mg} / \mathrm{kg}$ (TPO), and Intraperitonealy (TIP at $150 \mathrm{mg} / \mathrm{kg}$ ) in Comparison with the Effect of Proglumide $(135 \mathrm{mg} / \mathrm{kg}$ Subcutaneously: PRG). Control Animals Received Saline Orally (SPO) or Saline Intraperitonealy (SIP). Values in Brackets Represent Number of Animals and Data Represents mean \pm sem. ${ }^{* *} \mathbf{p}<0.001$ TPO versus TIP or PRG. * $\mathbf{p}=0.015$ TIP versus PRG.

\section{Discussion}

Our laboratory conduct studies to explore the anti-diabetic efficacy of herbs and plant parts mainly due to 3 reasons: 1 ) to investigate claims for several herbal and plant extracts that exist in the middle east and used by traditional healers to treat diabetes; 2) to identify the active ingredients that are responsible for the anti-diabetic effect; 3) to compare the plants in the area with those used in other parts of the world. In the last few decades, there was a renewed interest in herbal medicines and several potential antidiabetic plants have been studied to identify a wide array of chemically derived plant compounds for their possible diabetes therapy. Usually natural extracts can provide marked pharmacological actions with no or rare side effects if they were taken in the specified doses. Recently, Saravanamuttu and Sudarsanam (2012) reviewed anti-diabetic plants from different sources including some molecular docking studies on three plants to authenticate their affinity and therapeutic efficacy.

The present investigative study showed that the aqueous extracts of the aerial parts of Artemisia herba alba, phangnalon rupestre and Eryngium creticum produced significant hypoglycemic as well as antidiabetic activities in experimental animals while all the other plant extracts investigated failed to produce any hypoglycemic effect. In fact, some of the plant extracts produced significantly hyperglycemic effects following a glucose load such as myrtus communis and prosopis farcta (Twaij et al, 1987b; Twaij et al, 1989). Therefore, the later plant extracts results contrasted with the claimed hypoglycemic benefit of these as recommended by the local herbalists in folk medicine (Twaij et al, 1988a). Aqueous 
extracts and not organic solvent or other extracts are used in all of our studies. We think that this could be the cause behind their claimed beneficial application in herbal medicine and in addition, it seemed that the aqueous extracts had minimum or undiscovered adverse effects.

We have also demonstrated in this paper that the T. Polium aqueous extract had a healing effect on ulcers induced by reserpine in rats. This healing effect was more obvious after oral administration of the extract. The mechanism underlying this effect is not evident from the data available. Further investigations are needed, such as studying its effect on serum calcium, gastric acid secretion and intestinal motility. The extracts of other plants were briefly tested for antiulcer activity but none in our laboratory produced a significant effect. Future studies would now be justified trying to identify and isolate the active constituents responsible for the anti-diabetic and anti-ulcer activities.

\section{Acknowledgements}

The authors would like to thanks the staff of the Pharmacology Department and animal house at the Faculty of Pharmacy, Philadelphia University for their help and support in providing the lab space to conduct the experiments. The authors wish to declare no conflict of interest.

\section{References}

A-Jaleel, H. \& Twaij, H. (2002). 'Screening for a Possible Hypoglycemic Activity of Selected Jordanian Medicinal Plants,' Part I. Jordanian Journal for Applied Science,4: 1-7

AI-Khazarji, S. M., AI-Shamony, L. A. \& Twaij, H. A. A. (1993). "Hypoglycaemic Effect of. Artemisia Herba-Alba. Effect of Different Parts and Influence of the Solvent on Hypoglycaemic Activity," Journal of Ethnopharmacology. 40: 163-166.
Al-Shamaony, L., Al-Khazraji, S. M. \& Twaij, H. A. A. (1994). "Hypoglycaemic Effect of Artemisia Herba Alba. II. Effect of a Valuable Extract on Some Blood Parameters in Diabetic Animals," Journal of Ethnopharmacology 43: 167-171

Farnsworth, R. \& Segelman, A. B. (1971). 'Hypoglycemic Plants,' Volume 57, Tile \& Till: 52-56.

Lewis, H. W. \& Elvin-Lewis, M. P. H. (1977). 'Plant Botany: Plants Affecting Man's Health,' john Wiley and Sons, New York, pp.1, 36, 98, 218, 515.

Litchfield, Jr., J. T. \& Wilcoxon, F. (1949). “A Simplified Method of Evaluating Dose-Effect Experiments," Journal of. Pharmacology and Experimental Therapeutics 96; 99-113.

Said, M. (1969). 'Hamdard Pharmacopoeia of Eastern Medicine,' Hamadard National Foundation, Time press, Karachi, Pakistan, page 42 .

Saravanamuttu, S. \& Sudarsanam, D. (2012). "Antidiabetic Plants and their Active Ingredients: A Review," International Journal of Pharmaceutical Sciences \& Research 3(10); 3639-3650.

Twaij, H. A. A. \& Al-badr, A. A. (1988). "Hypoglycemic Activity of Artemisia Herba Alba," Elsevier Scientific Publishers Ireland. Journal of Ethnopharmacology 24: 123-126.

Twaij, H. A. A., Al-badr, A. A. \& Abul-Khail, A. (1987a). "Anti-Ulcer Activity of Teucrium Polium," International Journal of Crude Drug Research 25: 125-128.

Twaij, H. A. A. \& A-jaleel, H. (2002). 'Screening for a Possible Hypoglycemic Activity of Selected Jordanian Medicinal Plants,' Part II. Jordanian Journal for Applied Science, 4: 16-21. 
Twaij, H. A. A., Kerry, A., Al-jebory, A. A. \& Hammad, M. N. (1987b). 'Crataegus Azarolus Linn.Pharmacology and Phytochemistry,' Part I. Pharmacology J. Bio. Sci. Res, 18,105125.

Twaij, H. A. A., Kerry, A. \& Al-Khazraji, S. M. (1983). 'Some Pharmacological, Toxicological and Phytochemical Investigations on Centaurea Phylloceph,' Journal of Ethnopharmacology, 9 (1983) 29914299

Twaij, H. A. A., Mahmoud, S. N., \& Khalid, R. M. (1989). "Screening of Some Iraqi Medicinal Plants for their Molluscicidal Activities," Fitoterapia 1989 Vol. 60 No. 3 pp. 267-268

Twaij, H. A. A., Sayed-Ali, H. M. \& AlZohry, A. M. (1988a). "Pharmacological Phytochemical and Antimicrobial Studies on Myrtus Communis Part I. Cardiovascular and Phytochemical Studies," Journal of Biological Sciences Research, 19(1): 29-40.

Twaij, H. A. A., Sayed-Ali, H. M. \& AlZohry, A. M. (1988b). "Pharmacological Phytochemical and Antimicrobial Studies on Myrtus Communis Part 2. Glycemic and Antimicrobial Studies," Journal of Biological Sciences Research, 19(1): 41-52. 